\title{
CALIDAD MICROBIANA DE LA CARNE DE OVINO DERIVADA DE LA EDAD DE SACRIFICIO Y TIEMPO DE MADURACION
}

\section{MICROBIAL QUALITY OF SHEEP MEAT DERIVED FROM THE AGE OF SLAUGHTER AND RIPENING TIME}

\section{${ }^{1 *}$ Duran Osorio Daniel, ${ }^{1}$ Trujillo Navarro Yanine y ${ }^{1}$ Morales Ocampo Henry}

Universidad de Pamplona, Facultad de Ingenierías y Arquitectura, Departamento de Alimentos, Grupo de Investigación GINTAL, Grupo de Investigación en Bioingeniería Alimentaria. Ciudadela Universitaria Km 1 vía a Bucaramanga. Pamplona, Norte de Santander, Colombia. danielduran@unipamplona.edu.co

Recibido 15 de Marzo de 2017; Aceptado 15 de junio de 2017.

\section{RESUMEN}

Un índice que permite garantizar mayor seguridad sanitaria del consumo de la carne ovina en fresco es su calidad microbiana, que se debe alcanzar durante todo el proceso de producción y comercialización. En Colombia existen pocas plantas de sacrificio específicas y el sacrificio se hace a diferentes edades. Por ello, el propósito fue evaluar la calidad microbiológica de la carne ovina (corte rack) derivada de la edad de sacrificio y del tiempo de maduración. De canales de ovinos de 4, 6, 8 y 10 meses de edad y madurada en tres tiempos (24, 48 y 72 horas), se obtuvo el corte rack, siendo empacado al vacío (95\%) y almacenado durante 20 días a $4 \pm 2^{\circ} \mathrm{C}$. Se analizaron en los días $0 ; 10$ y 20 coliformes totales 
y fecales, Staphillococcus auereus, Clostridium sulfito reductoras, Salmonella y Listeria monocytogenes. Se obtuvo como resultados que durante el periodo de almacenamiento, todos los tratamientos relacionados con la edad del animal y la maduración de la carne, presentaron carga microbiana que cumple con los requisitos establecidos por el INVIMA. La carne procedente de animales de 10 meses de edad fue la que registró menores recuentos microbiológicos. Por tanto, la edad del animal y la maduración no es factor influyente en la microbiología analizada en la carne ovina.

Autor a quien dirigir la correspondencia: Duran Osorio Daniel $^{*}$ Correo electronico: danielduran@unipamplona.edu.co
Palabras clave: carga microbiana, carne de ovino, cordero, maduración, sacrificio

\section{ABSTRACT}

An index that allows to guarantee greater sanitary safety of the consumption of the ovine meat in fresh is its microbial quality, that must be achieved during the whole process of production and commercialization. In Colombia there are few specific slaughter plants and the sacrifice is made at different ages. Therefore, the purpose was to evaluate the microbiological quality of the ovine meat (court rack) derived from the age of slaughter and the ripening time. Of sheep carcases of $4,6,8$ and 10 months of age and repined in three times (24, 48 and 72 hours), the rack court was obtained, being packed in vacuum (95\%) and stored for 20 days at $4 \pm 2{ }^{\circ} \mathrm{C}$. were analyzed in days $0 ; 10$ and 20 total and fecal coliforms, Staphillococcus Auereus, Clostridium sulfite reducing, Salmonella and Listeria monocytogenes. It was obtained as results that during the period of storage, all the treatments related to the age of the animal and the ripening of the meat, presented microbial load that meets the requirements established by the INVIMA. The meat from animals of 10 months of age was the one that recorded lower microbiological 
counts. Therefore, the age of the animal and ripening is not an influential factor in the microbiology analyzed in the ovine meat.

Keywords: lamb, microbial load, sheep meat, ripening, slaughter

\section{INTRODUCCIÓN}

De acuerdo con los nuevos estilos de vida y los importantes cambios sociales que se vienen presentando, han adquirido en la industria cárnica singular importancia nuevos tipos de envase y empaque. Se destacan entre ellos las porciones individuales, los empaques institucionales, los materiales que permiten vidas de anaquel más largas y que reemplazan a los tradicionales, generalmente más costosos (Restrepo et al., 2001).

Los cambios físicos, químicos y microbiológicos que se producen en la carne fresca son estrictamente una función de la temperatura y la humedad. El control de la temperatura y la humedad constituye, consecuentemente, en la actualidad el método más importante de conservación de la carne para atenerse a las necesidades de los procedimientos o del comercio al por menor de los países industrialmente desarrollados del mundo y está siendo cada vez más empleado en las zonas urbanas, particularmente por parte de hoteles, abastecedores de comidas e instituciones hospitalarias de los países en desarrollo. Por ejemplo, el aumento de las bacterias se reduce a la mitad con cada descenso de la temperatura de $10{ }^{\circ} \mathrm{C}$ y prácticamente se detiene en el punto de congelación; es decir, la carne se conservará por lo menos el doble de tiempo a $0{ }^{\circ} \mathrm{C}$ que la carne con un nivel análogo de contaminación, pero conservada a $7^{\circ} \mathrm{C}$; o se conservará por lo menos cuatro veces más tiempo a $0{ }^{\circ} \mathrm{C}$ que ha $10^{\circ} \mathrm{C}$. La temperatura ideal de almacenamiento de la carne fresca oscila en torno al punto de congelación alrededor de $-1^{\circ} \mathrm{C}(\mathrm{FAO}, 1993)$.

La calidad de la carne ovina (la calidad fisicoquímica, reológica y sensorial) depende en gran medida de parámetros antes-morten (sistema productivo, raza, edad y peso al sacrificio) y de parámetros pos-morten especialmente en la conversión musculo a carne y en la maduración (Duran y Trujillo, 2014). Por otro lado, controlar la calidad microbiana durante todo el proceso de producción y comercialización es un índice que permite garantizar mayor seguridad sanitaria del consumo de la carne ovina en fresco y derivados. 
De otra parte, la refrigeración es el método más difundido para la conservación de la carne. El frío inhibe el desarrollo de los microorganismos que decrece hasta llegar a la temperatura a la cual se paraliza totalmente (Prändl et al., 1994). En la microbiología de la carne, las bacterias aerobias Gram negativas son de mayor importancia especialmente las del género Pseudomonas sspp., Moraxella spp., Acinetobacter spp., Flavobacterium spp., siendo las responsables de la alteración de la carne refrigerada (Nortje et al., 1990). Adicionalmente pueden existir bacterias productoras de ácido láctico, hongos, levaduras y virus entéricos en bajas cantidades. La contaminación es muy variable y pueden incluirse algunos microorganismos patógenos como Salmonella spp., Staphilococcus aureus, Yersinia enterolitica/pseudotuberculosis,
Campylobacter jejuni/coli, Listeria monocytogenes, Escherichia coli, Bacillus cereus, Clostridium perfringens y Clostridium botulinum, que provienen ya sea de la microflora intestinal o del medio ambiente.

Según Serrano (2014), para de extender la vida útil de la carne se han utilizado diferentes sistemas de envasado y de conservantes naturales. Así, el envasado al vacío o con atmósferas modificadas los más utilizados comercialmente, ya que éste es in indicador de higiene para el consumidor y una estrategia de comercialización mejorando la presentación del producto. Por lo anterior, la presente investigación se enmarca en evaluar la calidad microbiológica de la carne de ovino en el corte rack analizando la incidencia de la edad de sacrificio y el tiempo de maduración.

\section{MATERIALES Y MÉTODOS}

Fueron utilizados 36 ovinos (machos enteros) de raza criolla provenientes de la ecoregión de García Rovira (Santander). Tomando 9 animales de cada edad (4, 6, 8 y 10 meses). Sometidos los ovinos en ayuno de 12 horas, fueron sacrificados bajo condición higiénica sanitaria siguiendo los protocolos en la planta de cárnicos de la Universidad de Pamplona. Las canales fueron sometidas a tres tiempos $(24,48$ y 72 horas) de maduración, bajo condiciones de refrigeración $\left(4 \pm 2{ }^{\circ} \mathrm{C}\right)$. Posteriormente, se obtuvo el corte de rack (entre la $5^{\mathrm{a}}$ y $13^{\mathrm{a}}$ costilla), siendo empacados al vacío (95\%) y almacenados durante 20 días a $4 \pm 2{ }^{\circ} \mathrm{C}$. Se analizaron en los días $0 ; 10$ y 20 recuento de Coliformes por placa (totales y fecales) según NTC 4458:2007, Staphylococcus aureus. coagulasa positivo, según la NTC 4779:2007; recuento de esporas de Clostridium sulfio reductoras, mediante la 
NTC 4834:2000; detección de Salmonella spp. en $25 \mathrm{~g}$ de producto, según la NTC 4574:2007 y, finalmente, la detección de
Listeria monocytogenes en $25 \mathrm{~g}$ de producto, según lo dispuesto en la NTC 4666:1999.

\section{RESULTADOS Y DISCUSIÓN}

Los resultados microbiológicos de la carne (corte rack) de ovino según la edad al sacrificio y tiempo de maduración almacenados durante 20 días a $4 \stackrel{\circ}{\circ}$ (tabla
1), presentan una carga microbiana que se encuentra dentro de los requisitos mínimos establecidos por el INVIMA (1998) durante los 20 días de almacenamiento.

Tabla 1. Microbiología de la carne según la edad al sacrificio y tiempo de maduración

\begin{tabular}{|c|c|c|c|c|c|c|c|c|c|c|c|c|}
\hline \multicolumn{13}{|c|}{ CARNE DE ANIMALES DE 4 MESES DE EDAD } \\
\hline Día & \multicolumn{3}{|c|}{$\begin{array}{l}\text { Coliformes totales } \\
\qquad \text { (NMP) }\end{array}$} & \multicolumn{3}{|c|}{$\begin{array}{c}\text { Coliformes } \\
\text { fecales } \\
\text { (NMP) }\end{array}$} & \multicolumn{3}{|c|}{$\begin{array}{l}\text { S. aureus } \\
\log (\text { UFC/g) }\end{array}$} & \multicolumn{3}{|c|}{$\begin{array}{c}\text { Clostridium spp } \\
\text { sulfito reductor } \\
\\
\log (\mathrm{UFC} / \mathrm{g})\end{array}$} \\
\hline * & $24 h$ & $48 \mathrm{~h}$ & $72 \mathrm{~h}$ & $24 h$ & $48 \mathrm{~h}$ & $72 h$ & $24 \mathrm{~h}$ & $48 \mathrm{~h}$ & $72 \mathrm{~h}$ & $24 \mathrm{~h}$ & $48 \mathrm{~h}$ & $72 h$ \\
\hline 0 & 460 & 9 & 97 & 93 & 4 & 12 & $<1,48$ & $<1,48$ & $<1,48$ & $<1,48$ & $<1,48$ & $\begin{array}{c}<1,4 \\
8\end{array}$ \\
\hline 10 & 1100 & 1100 & 23 & 93 & 75 & $<3$ & $<1,48$ & $<1,48$ & $<1,48$ & $<1,48$ & $<1,48$ & $\begin{array}{c}<1,4 \\
8 \\
\end{array}$ \\
\hline 20 & $>2400$ & $>2400$ & $>2400$ & 670 & 42 & 780 & $<1,48$ & $<1,48$ & $<1,48$ & $<1,48$ & $<1,48$ & $\begin{array}{c}<1,4 \\
8 \\
\end{array}$ \\
\hline \multicolumn{13}{|c|}{ CARNE DE ANIMALES DE 6 MESES DE EDAD } \\
\hline * & $24 \mathrm{~h}$ & $48 \mathrm{~h}$ & $72 \mathrm{~h}$ & $24 h$ & $48 \mathrm{~h}$ & $72 \mathrm{~h}$ & $24 \mathrm{~h}$ & $48 \mathrm{~h}$ & $72 \mathrm{~h}$ & $24 \mathrm{~h}$ & $48 \mathrm{~h}$ & $72 h$ \\
\hline 0 & 20 & 93 & 93 & 20 & 4 & 93 & $<1,48$ & $<1,48$ & $<1,48$ & $<1,48$ & $<1,48$ & $\begin{array}{c}<1,4 \\
8\end{array}$ \\
\hline 10 & 93 & $>2400$ & 75 & 9 & 23 & 43 & $<1,48$ & $<1,48$ & $<1,48$ & $<1,48$ & $<1,48$ & $\begin{array}{c}<1,4 \\
8 \\
\end{array}$ \\
\hline 20 & $>2400$ & $>2400$ & $>2400$ & 240 & 240 & 240 & $<1,48$ & $<1,48$ & $<1,48$ & $<1,48$ & $<1,48$ & $\begin{array}{c}<1,4 \\
8 \\
\end{array}$ \\
\hline \multicolumn{13}{|c|}{ CARNE DE ANIMALES DE 8 MESES DE EDAD } \\
\hline * & $24 \mathrm{~h}$ & $48 \mathrm{~h}$ & $72 \mathrm{~h}$ & $24 h$ & $48 \mathrm{~h}$ & $72 \mathrm{~h}$ & $24 \mathrm{~h}$ & $48 \mathrm{~h}$ & $72 \mathrm{~h}$ & $24 \mathrm{~h}$ & $48 \mathrm{~h}$ & $72 h$ \\
\hline 0 & 43 & $<2400$ & 150 & $>3$ & 240 & 150 & $<1,48$ & $<1,48$ & $<1,48$ & $<1,48$ & $<1,48$ & $\begin{array}{c}<1,4 \\
8\end{array}$ \\
\hline 10 & 93 & $<2400$ & $<2400$ & 43 & 460 & 240 & $<1,48$ & $<1,48$ & $<1,48$ & $<1,48$ & $<1,48$ & $\begin{array}{c}<1,4 \\
8\end{array}$ \\
\hline 20 & 93 & $<2400$ & 460 & 23 & 150 & 43 & $<1,48$ & $<1,48$ & $<1,48$ & $<1,48$ & $<1,48$ & $\begin{array}{c}<1,4 \\
8\end{array}$ \\
\hline
\end{tabular}


Continuación de la tabla 1.

\begin{tabular}{|c|c|c|c|c|c|c|c|c|c|c|c|c|}
\hline Día & \multicolumn{3}{|c|}{$\begin{array}{l}\text { Coliformes totales } \\
\qquad \text { (NMP) }\end{array}$} & \multicolumn{3}{|c|}{$\begin{array}{c}\text { Coliformes } \\
\text { fecales } \\
\text { (NMP) }\end{array}$} & \multicolumn{3}{|c|}{$\begin{array}{l}\text { S. aureus } \\
\log (\text { UFC/g) }\end{array}$} & \multicolumn{3}{|c|}{$\begin{array}{c}\text { Clostridium spp } \\
\text { sulfito reductor } \\
\\
\log (\mathrm{UFC} / \mathrm{g})\end{array}$} \\
\hline \multicolumn{13}{|c|}{ CARNE DE ANIMALES DE 10 MESES DE EDAD } \\
\hline * & $24 \mathrm{~h}$ & $48 \mathrm{~h}$ & $72 \mathrm{~h}$ & $24 \mathrm{~h}$ & $48 h$ & $72 \mathrm{~h}$ & $24 \mathrm{~h}$ & $48 \mathrm{~h}$ & $72 \mathrm{~h}$ & $24 \mathrm{~h}$ & $48 \mathrm{~h}$ & $72 \mathrm{~h}$ \\
\hline 0 & $>2400$ & 4 & $<3$ & 4 & $<3$ & $<3$ & $<1,48$ & $<1,48$ & $<1,48$ & $<1,48$ & $<1,48$ & $\begin{array}{c}<1,4 \\
8\end{array}$ \\
\hline 10 & $>2400$ & 460 & $<3$ & 93 & 460 & $<3$ & $<1,48$ & $<1,48$ & $<1,48$ & $<1,48$ & $<1,48$ & $\begin{array}{c}<1,4 \\
8\end{array}$ \\
\hline 20 & $>2400$ & 4 & $<3$ & 93 & 4 & $<3$ & $<1,48$ & $<1,48$ & $<1,48$ & $<1,48$ & $<1,48$ & $\begin{array}{c}<1,4 \\
8\end{array}$ \\
\hline \multicolumn{4}{|c|}{ INVIMA 1998 (límite máximo) } & \multicolumn{3}{|c|}{ 120-1100 NMP } & \multicolumn{3}{|c|}{2 - 3 ufc/g } & \multicolumn{3}{|c|}{$2-3$ ufc/g } \\
\hline
\end{tabular}

* Tiempo de maduración

La salmonella, es un agente considerado por el hombre patógeno, que no pertenece a la población microbiana normal. Este género puede causar diferentes síntomas que van desde ligeras gastroenteritis a enfermedades sistémicas complicadas (Cox, 2000; Bezirtzouglou et al. 2000). De acuerdo a lo anterior, los valores de los recuentos de Salmonella y Listeria monocytogenes fueron negativo (no presencia) en todos los tratamientos (edad del animal y tiempo de maduración). Por consiguiente, se muestra así la calidad sanitaria y el buen manejo desde el sacrificio, pasando por la maduración en canal y por el corte desarrollado durante la preparación del Rack, a diferencia de Espineles (2012) quien evaluó la calidad microbiológica en carne de ovino y caprina sometida a un proceso de salado industrial y se evidenció la presencia de coliformes fecales y clostridios sulfitoredutores en algunos lotes lo cual es una posible deficiencia en las buenas prácticas de fabricación a lo largo del proceso de transformación o materia prima, observando también la ausencia de Salmonella, S. aureus cuagulasa positivos, coliformes fecales y clostridios sulfito-reductores, están dentro de los valores encontrados en la literatura, asegurando que estos productos poseen un riesgo mínimo de provocar intoxicaciones alimentarias debido a estos microorganismos.

La presencia de coliformes esta correlacionado con contaminación fecal. Este tipo de indicadores son aplicados en la industria de alimentos. Como estos no son 
solo encontrados en las heces, la presencia de coliformes totales, no es indicativo de contaminación fecal, necesariamente. (Franco y Landgraf, 1996). En este sentido, en lo que concierne a los coliformes fecales y totales es variable según la edad al sacrificio de animal y el tiempo de maduración, con una tendencia a aumentar con relación al tiempo de almacenamiento. (Trujillo et al., 2014; Trujillo et al., 2015). Asimismo, en la tabla 1 se observa también que la carne procedente de los animales de 10 meses de edad y a los tres tiempos de maduración es donde se muestran los valores más bajos en los recuentos de coliformes fecales, indicando que este tratamiento sería el mejor para mantener la microbiología inicial durante el almacenamiento. Estos resultados pueden deberse, al correlacionar los recuentos con variables fisicoquímicas datos no mostrados según Duran y Trujillo (2014), en donde a mayor edad de animal la carne exhibe mayor contenido de proteína, grasa y cenizas pero menor contenido capacidad de retención de agua (CRA) y contenido de humedad. Por otro lado, el recuento de Staphylococcus aureus y Clostridium sulfio reductoras, se mantienen durante el tiempo de almacenamiento a $4^{\circ} \mathrm{C}$ y no es afectado por la edad del animal al sacrificio y el tiempo de maduración.

\section{CONCLUSIONES}

Los recuentos de Salmonella fueron negativos en todos los tratamientos y al ser considerada como un agente patógeno, se pudo demostrar la calidad sanitaria y el buen manejo desde el sacrificio durante todo el procesado y almacenamiento.

En la carne de ovino (corte rack) empacada al vacío se conservó que durante 20 días bajo condiciones de refrigeración presentó una carga microbiana que cumple con los requisitos establecidos por el INVIMA en todos los tratamientos.

La edad del animal y el tiempo de maduración no es factor influyente en la microbiología analizada, Siendo la carne procedente de animales de 10 meses de edad la que registró los menores recuentos microbiológicos. 


\section{REFERENCIAS BIBLIOGRAFICAS}

Bezirtzouglou, E.; Maipa, V.; Voidarou, C.;

Tsiotsais, A. \& Papapetropoulou, M. (2000).

Food-borne intestinal bacterial pathogens.

Microbial Ecology in Health and Disease.

Suppl 2, pp. 96-104.

Cox, J. (2000). Salmonella. Introduction. In:

Robinson, R.; Batt, C. \& Patel, P. Encyclopedia of food microbiology. Volume

3. Bath: Academic Press. ISBN 0-12227070-3. pp. 1928-1937.

Duran, D. S. y Trujillo, N. Y. (2014). Parametrización cualitativa y cuantitativa de la calidad en la producción cárnica ovina en base en la edad y del peso al sacrificio. Pamplona (Colombia): Editorial Sello editorial Unipamplona.

Espineles, KP (2012). Análisis microbiológico para control cualitativo de carne ovina y caprina, seca y salada. Trabajo de Maestría. Instituto Politécnico de Bragança.

FAO. (1993). Estructura y funcionamiento de mataderos medianos en países en desarrollo.

http://www.fao.org/docrep/004/t0566s/T05 66S12.htm\#ch12.2. (Consultado diciembre 2013)
Franco, B. D. y Landgraf, M. (2003). Microbiologia dos alimentos. São Paulo: Atheneu, $182 \mathrm{p}$.

Instituto nacional de vigilancia de medicamentos y alimentos (INVIMA) (1998), Manual de técnicas de análisis para control de calidad microbiológico de alimentos para consumo humano. Bogotá (Colombia): INVIMA.

Instituto Colombiano de normas técnicas y certificación INCONTEC, 1999, Método horizontal para la detección de Listeria monocytogenes NTC 4666, Bogotá, Colombia.

Instituto Colombiano de normas técnicas y certificación INCONTEC, 2000, Método horizontal para el recuento de Clostridium sulfito reductores e identifiación de Clostridium perfringens. Técnica de recuento de colonias NTC 4834, Bogotá, Colombia.

Instituto Colombiano de normas técnicas y certificación INCONTEC, 2007, Método horizontal para el recuento de estafiicocos coagulasa positiva (Staphylococcus aureus y otras especies) NTC 4779, Bogotá, Colombia. 
Instituto Colombiano de normas técnicas y certificación INCONTEC, 2007, Método horizontal para la detección de Salmonellas spp NTC 4574, Bogotá, Colombia.

Instituto Colombiano de normas técnicas y certificación INCONTEC, 2007, Recuento de coliformes totales NTC 4458, Bogotá, Colombia.

Nortje, GL., Nel, L., Jordaan, E., Badenhorst, K., Goed-hart, G., Holzapfel WH. y Grimbeek RJ. (1994). “A quantitative survey of meat production chain to determine the microbial profile of the final product", J Food Protect, 53, pp 411-417.

Prändl, O., Fischer, A., Schmidhofer, T. y Sinell, HJ. (1994). Tecnología e Higiene de la Carne. Zaragoza (España): Editorial Acribia.

Restrepo, D. Arango, C. Restrepo, R y Campuzano, A. (2001). Industria de carnes. Editorial Universidad Nacional de Colombia.

Serrano, E. R. (2014). Extensión de la vida comercial de la carne de cordero mediante suplementación dietética con extracto de romero. Tesis Doctoral. Universidad de Murcia.

Trujillo N., Yanine, Duran O., Daniel y Loturco H. Anabelly. (2014). Influencia de la castración, ecorregión y época del año en la calidad sensorial de la carne de ovejo. Revista @limentech, Ciencia y Tecnología Alimentaria. ISSN 1692-7125. Volumen 12 No 1. Pp: $23-31$.

Trujillo, N. Yanine, Ayala M. Magda, Duran O. Daniel S. (2015). Influencia de la edad y el tiempo de madurez en la capacidad de retención de agua (CRA) en la carne de ovino criollo. Revista @limentech, Ciencia y Tecnología Alimentaria. ISSN 1692-7125. Volumen $13 \mathrm{~N}^{\circ} 1 . \mathrm{Pp}: 41-47$. 